\title{
A mathematical space mapping model for ballistic carbon nanotube field-effect transistors
}

\author{
Farnousha Emamifar $^{1} \cdot$ Reza Yousefi $^{1}$
}

Received: 28 January 2015/ Accepted: 15 September 2015/Published online: 11 November 2015

(C) The Author(s) 2015. This article is published with open access at Springerlink.com

\begin{abstract}
In this study, a mathematical model is presented based on mathematical space mapping for ballistic carbon nanotube field-effect transistors. This model is generalized from another model that was based on the concept of neural space mapping to calculate the three parameters of a coarse model. These parameters were the threshold voltage, the Early voltage, and assumed constant $k$ of a modified "level 1" MOSFET model in simulation program with integrated circuit emphasis (SPICE). In this work, three analytical relations are introduced to replace the neural networks of the main model. The comparisons between the proposed model and a well-known reference model, named FETToy, show that the proposed model had reasonable accuracy in terms of different biases and physical parameters.
\end{abstract}

Keywords Carbon nanotube $\cdot$ Field-effect transistor . Modeling $\cdot$ FETToy

\section{Introduction}

Having an accurate and fast model to be used in device simulators is one of the future challenges for simulating carbon nanotube metal-oxide-semiconductor (MOS) transistors. In this regard, there have been many works, most of which have paid attention to ballistic conditions [1-3] and

Reza Yousefi

r.yousefi@iaunour.ac.ir

Farnousha Emamifar

farnousha.emamifar@gmail.com

1 Department of Electrical Engineering, Nour Branch, Islamic Azad University, Nour, Iran some have considered non-ballistic conditions as well $[4,5]$. Since the mean free path of electrons moving at carbon nanotubes is about 0.6 micron, which is much more than the usual channel length of carbon nanotube transistors, the assumption of ballistic transport is usually used in these transistors [6].

One of the reference works for modeling ballistic conditions in MOSFET is FETToy model [7], which is a numerical model for MOSFET simulation in ballistic conditions. Unfortunately, because of numerical computation requirement and existence of the self-consistent loop, they are not suitable for use in many device simulators. Therefore, so many works have been done to delete computational requirements.

In a number of modified models, analytical mathematical model [1, 8], models based on neural networks (NNs) [10] or models based on neural space mapping (NSM) [9, 12] have been proposed for the direct calculation of output current. In [11], a circuit model was presented that could implement the FETToy model in the device simulators if an analytical solution is available for its charge integral equations.

Because of the need for only an analytical model for the charge integral equation, such kind of implementation will be more accurate than the models which directly present an analytical solution for current calculations. So, a bunch of refinements has been proposed to provide analytical relations for calculating the charge integral [3].

The NSM concept can be used to obtain a model for the new devices quickly. This allows us to change the model of the existing devices so that the resulting model can be used for new devices. The model presented in Ref. [9] is one of the models used this concept. In this model, a well-known "level 1" MOSFET model in SPICE [13] is modified by three neural networks such that its output would be equal to 
the one from FETToy. Three neural networks have been applied for predicting the parameters $V_{\mathrm{A}}, k$, and $V_{\mathrm{TH}}$, which are Early voltage, a model parameter, and threshold voltage, respectively. In this study, using the equation presented in [1] for the potential barrier at the top of the channel, some mathematical functions were proposed which could be used to calculate the fitted parameters $V_{\mathrm{A}}, k$, and $V_{\mathrm{TH}}$ as the alternative for the neural networks. The results obtained from the simulation and comparison with those of FETToy model demonstrated that the proposed model had acceptable and appropriate accuracy for different values of physical parameters and different bias conditions.

\section{Proposed model}

FETToy is a reference model for MOS-like carbon nanotube FET in the ballistic limit. It is based on the calculation of the charge and the voltage at the top of channel potential barrier in a self-consistent manner, which will be explained later in more detail. The device structure used in this work and some important parameters considered in FETToy are illustrated in Fig. 1. As mentioned above, need to numerical calculations is an important drawback of FETToy.

Using NSM concept, a model has been introduced in Ref. [9] to solve this problem. In this model, three neural networks have been applied for predicting the parameters $V_{\mathrm{A}}, k$, and $V_{\mathrm{TH}}$ of a well-known "level 1" MOSFET model in SPICE such that its output would be equal to the one of FETToy.

In this study, a mathematical model of the parameters $k$, $V_{\mathrm{A}}$, and $V_{\mathrm{TH}}$ was proposed to replace the neural networks used for calculating these parameters in a model introduced in Ref. [9].

In this reference, as mentioned before, in contrast to the conventional use of neural network, a neural space mapping concept was used to predict parameters of a coarse model, which was "level 1" of MOSFET model in SPICE, so that the output of the resulting model was the same as that obtained by FETToy.

In the proposed model, by using the equation for the charge density at the top of channel potential barrier introduced in Ref. [1], three mathematical models were presented for calculating the $V_{\mathrm{TH}}, V_{\mathrm{A}}$, and $k$. Then, by providing a mathematic model of suitability parameters $k$, $V_{\mathrm{A}}$, and $V_{\mathrm{TH}}$, a complete mathematical model is presented. The procedures are given in the following.

\section{$\mathbf{V}_{\text {th }}$}

From Ref. [1], the carrier density at the top of barrier injected from source can be modeled by following equation:
Fig. 1 Schematic of the device structure used in this work and an illustration of some important parameters used in FETToy. The green curve is the mid-gap energy band diagram of the transistor from the source to the drain

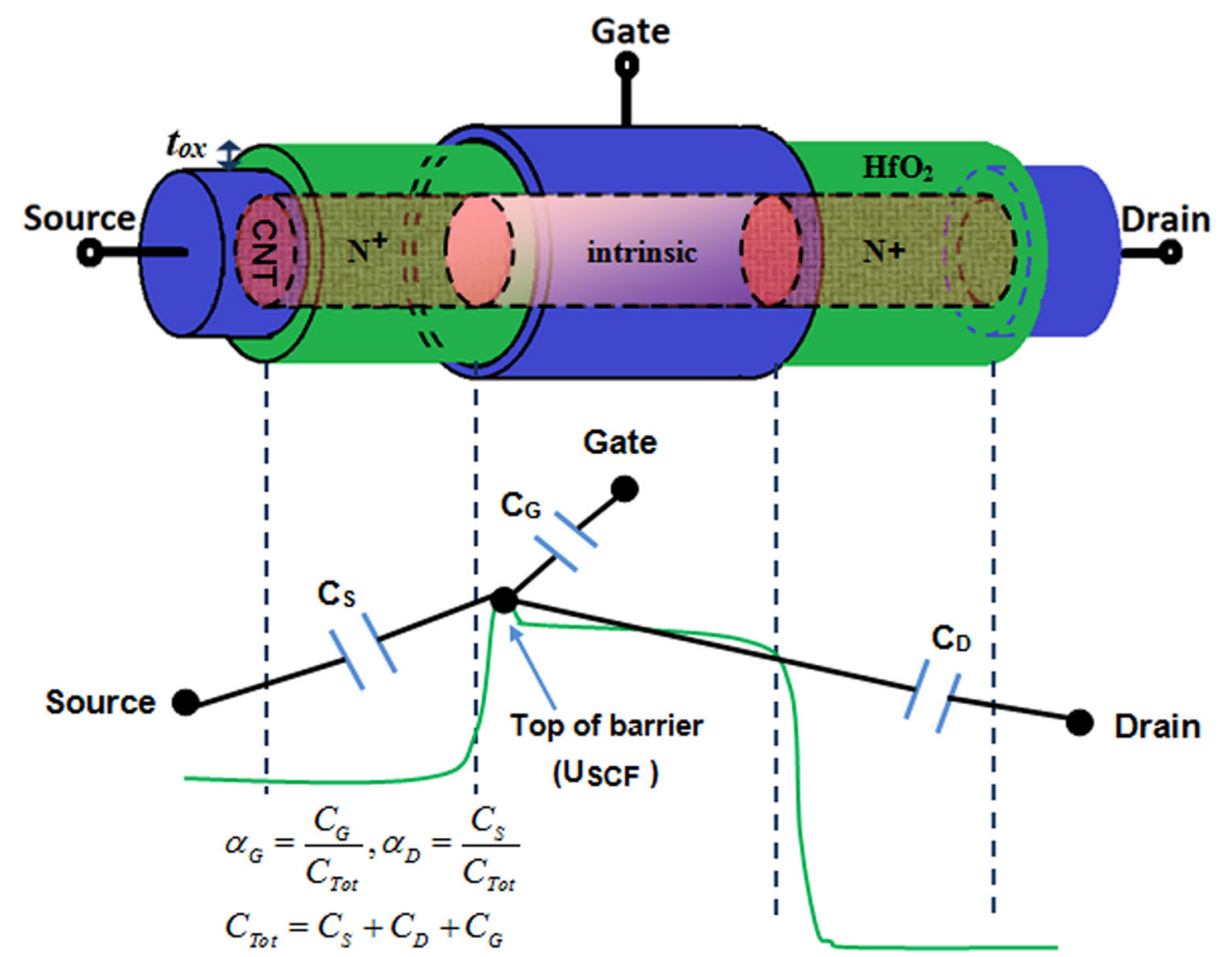




$$
\begin{aligned}
& N_{1}\left(U_{\mathrm{SCF}}\right)=\left\{\begin{array}{lc}
m_{1}^{2} U_{\mathrm{SCF}}+n_{1}^{2}, & U_{S C F} \leq E_{F}+2 k_{1 B} T \ln (\varepsilon) \\
m_{1}^{1} U_{\mathrm{SCF}}+n_{1}^{1}, & E_{F}+k_{B} T \ln (\varepsilon)<U_{S C F} \leq-\frac{n_{1}^{1}}{m_{1}^{1}} \\
0, & U_{S C F}>-\frac{n_{1}^{1}}{m_{1}^{1}}
\end{array}\right. \\
& \left\{\begin{array}{l}
m_{1}^{1}=D_{\circ} / 2\left(\begin{array}{c}
4 \sqrt{\left(E_{\mathrm{B}}-K_{\mathrm{B}} T \ln (\varepsilon)^{2}-E_{\mathrm{B}}^{2}\right.} \\
+\frac{\sqrt{E_{\mathrm{B}}-K_{\mathrm{B}} T \ln (\varepsilon)^{2}-E_{\mathrm{B}}^{2}}}{8 k_{\mathrm{B}} T}
\end{array}\right) \\
m_{1}^{2}=-D_{\circ} / 2 \\
n_{1}^{1}=\frac{D_{\circ} \sqrt{\left.E_{\mathrm{B}}-K_{\mathrm{B}} T \ln (\varepsilon)\right]^{2}-E_{\mathrm{B}}^{2}}-m_{1}^{1} E_{F}}{\left(m_{1}^{1}-m_{1}^{2}\right)\left(E_{\mathrm{F}}^{8}+2 K_{\mathrm{B}} T \ln (\varepsilon)\right]-n_{1}^{1}}
\end{array}\right.
\end{aligned}
$$

where $U_{\mathrm{SCF}}$ is the potential energy at the top of the barrier, according to FETToy model given by:

$$
\begin{aligned}
U_{\mathrm{SCF}}= & -q \alpha_{\mathrm{G}} V_{\mathrm{GS}}-q \alpha_{\mathrm{D}} V_{\mathrm{DS}}+\frac{q^{2}}{C_{\mathrm{Tot}}} \times\left(N_{1}\left(U_{\mathrm{SCF}}\right)\right. \\
& \left.+N_{2}\left(U_{\mathrm{SCF}}\right)+N_{0}\right)
\end{aligned}
$$

where $\alpha_{\mathrm{G}}$ and $\alpha_{\mathrm{D}}$ are parameters for gate and drain control on the channel potential $C_{\text {Tot }}$ is the total capacitance of transistor, $N_{1}$ and $N_{2}$ are charge densities injected into the channel (from source and drain), and $N_{O}$ is the mobile charge inherently present in the channel. Because of the small value of $N_{O}$, it was not considered in this study.

According to FETToy model [7], we have:

$$
\begin{aligned}
I= & I_{0} \underbrace{\left[n \left(1+\exp \left(\frac{E_{\mathrm{F}}-U_{\mathrm{SCF}}}{K_{\mathrm{B}} T}\right)\right.\right.}_{(1)} \\
& -\underbrace{\left.\operatorname{Ln}\left(1+\exp \left(\frac{E_{\mathrm{F}}-U_{\mathrm{SCF}}-q V_{\mathrm{DS}}}{K_{\mathrm{B}} T}\right)\right)\right]}_{(2)}
\end{aligned}
$$

where $q, k_{\mathrm{B}}, T, h$, and $E_{\mathrm{F}}$ are electronic charge, the Boltzmann constant, temperature, Planck's constant, and source Fermi energy level, respectively.

$V_{\text {DSsat }}$ is the saturation drain-source voltage. In this voltage, the dependence of output current on drain-source voltage reduces. Therefore, the second term can be neglected in the first sentence, as a result:

$\exp \left(\frac{E_{\mathrm{F}}-U_{\mathrm{SCF}}-q V_{\mathrm{DSsat}}}{K_{\mathrm{B}} T}\right) \ll 1$

For the sample equal to 0.01 , the following can be written:

$$
\begin{aligned}
& \exp \left(\frac{E_{\mathrm{F}}-U_{\mathrm{SCF}}-q V_{\mathrm{DSsat}}}{K_{\mathrm{B}} T}\right)=0.01 \\
& \Rightarrow E_{\mathrm{F}}-U_{\mathrm{SCF}}-q V_{\mathrm{DSsat}}=K_{\mathrm{B}} T \ln (0.01)
\end{aligned}
$$

In this case, $N_{2}\left(U_{\mathrm{SCF}}\right)$, i.e., the charge density at top of barrier injected from drain, can be neglected; thus:

$\left.N_{2}\left(U_{\mathrm{SCF}}\right)\right|_{V_{\mathrm{DSsat}}}=0$

On the other hand, if we rearranged the relationship $N_{1}\left(U_{\mathrm{SCF}}\right)$ based on the gate-source voltage, for the first interval of Eq. (1), we would have:

$$
\begin{aligned}
U_{\mathrm{SCF}} \leq & E_{\mathrm{F}}+2 k_{\mathrm{B}} \ln (\varepsilon) \\
\Rightarrow & -q\left(V_{\mathrm{GS}} \alpha_{\mathrm{G}}+V_{\mathrm{DS}} \alpha_{\mathrm{D}}\right)+q^{2} / c_{\mathrm{Tot}}\left(N_{1}\left(U_{\mathrm{SCF}}\right)\right. \\
& \left.+N_{2}\left(U_{\mathrm{SCF}}\right)-N_{\circ}\right) \leq E_{\mathrm{F}}+2 K_{\mathrm{B}} T \ln (\varepsilon) \\
\Rightarrow & V_{\mathrm{GS}} \geq \frac{-q \alpha_{\mathrm{D}} V_{\mathrm{DSsat}}-E_{\mathrm{f}}-2 K_{\mathrm{B}} T \ln (\varepsilon)+\left(q^{2} / c_{\mathrm{Tot}}\right) N_{1}\left(U_{\mathrm{SCF}}\right)}{q \alpha_{\mathrm{G}}}
\end{aligned}
$$

In this regard, for the second and third intervals, the following can be presented:

$$
\begin{aligned}
& N_{1}\left(U_{\mathrm{SCF}}\right)=\left\{\begin{array}{l}
m_{1}^{2} U_{\mathrm{SCF}}+n_{1}^{2}=N_{11} V_{\mathrm{GS}}>V_{\mathrm{GS} 1} \\
m_{1}^{1} U_{\mathrm{SCF}}+n_{1}^{1}=N_{11} V_{\mathrm{GS} 2} \leq V_{\mathrm{GS}}<V_{\mathrm{GS} 1} \\
0=N_{13} V_{\mathrm{GS}}<V_{\mathrm{GS} 2}
\end{array}\right. \\
& V_{\mathrm{GS} 1}=\left(-q \alpha_{\mathrm{D}} V_{\mathrm{DSsat}}-E_{\mathrm{F}}-2 K_{\mathrm{B}} T \ln (\varepsilon)+\frac{q^{2} N_{12}}{C_{\mathrm{Tot}}}\right) /\left(q \alpha_{\mathrm{G}}\right) \\
& V_{\mathrm{GS} 2}=\left(-q \alpha_{\mathrm{D}} V_{\mathrm{DSsat}}-\frac{n_{1}^{1}}{m_{1}^{1}}\right) /\left(q \alpha_{\mathrm{G}}\right) .
\end{aligned}
$$

Examinations showed that the desired values of $V_{\mathrm{GS}}$ are smaller than $V_{\mathrm{GS} 1}$, and as a result, the first interval can be ignored. According to the trivial amount of current in $V_{\mathrm{GS}}<V_{\mathrm{GS} 2}$, the second interval is the most important region. Using $U_{\mathrm{SCF}}$ in this region, $V_{\mathrm{DSsat}}$ can be obtained as follows.

By combining Eqs. (8) and (2), the value of $U_{\mathrm{SCF}}$ is achieved in $V_{D S}=V_{D S s a t}$ :

$$
\begin{aligned}
U_{\mathrm{SCFsat}} & =-q \alpha_{\mathrm{G}} V_{\mathrm{GS}}-q \alpha_{\mathrm{D}} V_{\mathrm{DSsat}}+\frac{q^{2}}{C_{\text {Tot }}}\left(m_{1}^{1} U_{\mathrm{SCFsat}}+n_{1}^{1}\right) \\
& =\left(-q \alpha_{\mathrm{G}} V_{\mathrm{GS}}-q \alpha_{\mathrm{D}} V_{\mathrm{DSsat}}+\frac{q^{2}}{C_{\mathrm{Tot}}} n_{1}^{1}\right) /\left(1-\frac{q^{2}}{C_{\mathrm{Tot}}} m_{1}^{1}\right)
\end{aligned}
$$

By inserting Eq. (9) in Eq. (5), the value of $V_{\mathrm{DSsat}}$ is achieved:

$$
V_{\mathrm{DSsat}}=\frac{\left(E_{\mathrm{F}}-K_{\mathrm{B}} T \ln (0.01)\right)\left(1-\frac{q^{2}}{C_{\mathrm{Tot}}} m_{1}^{1}\right)+q \alpha_{\mathrm{G}} V_{\mathrm{GS}}-q^{2} \frac{n_{1}^{1}}{C_{\mathrm{Tot}}} .}{q\left(1-\frac{q^{2}}{C_{\mathrm{Tot}}} m_{1}^{1}-\alpha_{\mathrm{D}}\right)} .
$$

According to the definition of $V_{\mathrm{DSsat}}=V_{\mathrm{GS}}-V_{\mathrm{TH}}$, the threshold voltage is computable from this relation: 
$V_{\mathrm{TH}}=V_{\mathrm{GS}}-\frac{\left(E_{\mathrm{F}}-K_{\mathrm{B}} T \ln (0.01)\right)\left(1-\frac{q^{2}}{C_{\mathrm{Tot}}} m_{1}^{1}\right)+q \alpha_{\mathrm{G}} V_{\mathrm{GS}}-q^{2} \frac{n_{1}^{1}}{C_{\mathrm{Tot}}}}{q\left(1-\frac{q^{2}}{C_{\mathrm{Tot}}} m_{1}^{1}-\alpha_{\mathrm{D}}\right)}$

\section{$k$ and $V_{A}$}

Now, we are going to calculate the two other parameters, $k$ and $V_{\mathrm{A}}$. In what continues, only $V_{\mathrm{DS}} \geq V_{\mathrm{DSs} a t}$ is taken into account; as a result, Eq. (3) is simplified as follows:

$I=I_{0} \times \operatorname{Ln}\left(1+\exp \left(\frac{E_{\mathrm{F}}-U_{\mathrm{SCF}}}{K_{\mathrm{B}} T}\right)\right)$

In this regard, we obtain current in two assumed points $V_{\mathrm{DS} 1}$ and $V_{\mathrm{DS} 2}=V_{\mathrm{DS} 1}+0.01$. For this purpose, from relations (2) and (8) for $V_{\mathrm{DS}} \geq V_{\mathrm{DSsat}}$, we have:

$U_{\mathrm{SCF}}=\left\{\begin{array}{cc}\frac{-q \alpha_{\mathrm{G}} V_{\mathrm{GS}}-q \alpha_{\mathrm{D}} V_{\mathrm{DS}}+\frac{q^{2}}{C_{\mathrm{Tot}}} n_{1}^{1}}{1-\frac{q^{2}}{C_{\mathrm{Tot}}} m_{1}^{1}}, & V_{\mathrm{GS}} \geq V_{\mathrm{GS} 2} \\ -q \alpha_{\mathrm{G}} V_{\mathrm{GS}}-q \alpha_{\mathrm{D}} V_{\mathrm{DS}}, & V_{\mathrm{GS}}<V_{\mathrm{GS} 2} .\end{array}\right.$

Therefore:

$$
\begin{array}{r}
V_{\mathrm{DS}}=V_{\mathrm{DS} 1} \Rightarrow U_{\mathrm{SCF} 1}=\left\{\begin{array}{rr}
U_{\mathrm{SCF} 11} & V_{\mathrm{GS}} \geq V_{\mathrm{GS} 2} \\
U_{\mathrm{SCF} 12} & V_{\mathrm{GS}}<V_{\mathrm{GS} 2} \\
\Rightarrow I_{1}=I\left(U_{\mathrm{SCF} 1}\right)
\end{array}\right. \\
V_{\mathrm{DS}}=V_{\mathrm{DS} 2} \Rightarrow U_{\mathrm{SCF} 2}=\left\{\begin{array}{rr}
U_{\mathrm{SCF} 21} & V_{\mathrm{GS}} \geq V_{\mathrm{GS} 2} \\
U_{\mathrm{SCF} 22} & V_{\mathrm{GS}}<V_{\mathrm{GS} 2} \\
\Rightarrow I_{2}=I\left(U_{\mathrm{SCF} 2}\right)
\end{array}\right.
\end{array}
$$

To eliminate the above-mentioned intervals, the following auxiliary function can be used:

$f_{1}=\frac{1}{1+\exp \left(-10 \frac{V_{\mathrm{GS}}-V_{\mathrm{GS} 2}}{K_{\mathrm{B}} T / q}\right)}$.

By using this function, we have:

$$
\begin{gathered}
U_{\mathrm{SCF}_{1}}=U_{\mathrm{SCF} 11} \times f_{1}+U_{\mathrm{SCF}_{12}} \times\left(1-f_{1}\right) \\
U_{\mathrm{SCF}_{2}}=U_{\mathrm{SCF}_{21}} \times f_{1}+U_{\mathrm{SCF}_{22}} \times\left(1-f_{1}\right) .
\end{gathered}
$$

The relations can be summarized as:

$$
\left\{\begin{array}{l}
I_{1,2}=I_{0} \operatorname{Ln}\left(1+\exp \left(\frac{E_{\mathrm{F}}-U_{\mathrm{SCF} 1,2}}{K_{\mathrm{B}} T}\right)\right) \\
U_{\mathrm{SCF}_{11,21}}=\frac{-q \alpha_{\mathrm{G}} V_{\mathrm{GS}}-q \alpha_{\mathrm{D}} V_{\mathrm{DS} 1,2}+\frac{q^{2}}{C_{\mathrm{Tot}}} n_{1}^{1}}{1-\frac{q^{2}}{C_{\mathrm{Tot}}} m_{1}^{1}} \\
U_{\mathrm{SCF}_{12,22}}=-q \alpha_{\mathrm{G}} V_{\mathrm{GS}}-q \alpha_{\mathrm{D}} V_{\mathrm{DS}_{1,2}} .
\end{array}\right.
$$

Then, considering the relation in NSM model in the saturation region, we have [9]:

$I_{\mathrm{DS}}=k V_{\mathrm{DSsat}}^{2}\left(1+\frac{V_{\mathrm{DS}}-V_{\mathrm{DSsat}}}{V_{A}}\right)$.

Using this relation for $V_{\mathrm{DS} 1}$ and $V_{\mathrm{DS} 1}+0.01$, the following can be obtained:

$$
\begin{aligned}
& I_{1}=k V_{\mathrm{DSsat}}^{2}\left(1+\frac{V_{\mathrm{DS} 1}-V_{\mathrm{DSsat}}}{V_{\mathrm{A}}}\right) \\
& I_{2}=k V_{\mathrm{DSsat}}^{2}\left(1+\frac{V_{\mathrm{DS} 1}+0.01-V_{\mathrm{DSsat}}}{V_{A}}\right) .
\end{aligned}
$$

From these two relations and by putting the current value using Eq. (12), the values of $k$ and $V_{A}$ are computed as:

$V_{\mathrm{A}}=\frac{I_{1}\left(V_{\mathrm{DS} 1}-V_{\mathrm{DSsat}}\right)-I_{2}\left(V_{\mathrm{DS} 1}+0.01-V_{\mathrm{DSsat}}\right)}{I_{2}-I_{1}}$

$k=\frac{\left(I_{1}-I_{2}\right) V_{A}}{V_{\text {DSsat }}^{2} \times 0.01}$.

The resulting model for $V_{\mathrm{TH}}, V_{\mathrm{A}}$, and $k$ is summarized below:

$$
\begin{aligned}
& \left\{\begin{array}{l}
V_{\mathrm{DSsat}}=\frac{\left(E_{\mathrm{F}}-K_{\mathrm{B}} T \operatorname{Ln}(0.01)\right)\left(1-\frac{q^{2}}{C_{\mathrm{Tot}}} m_{1}^{1}\right)+q \alpha_{\mathrm{G}} V_{\mathrm{GS}}-q^{2} \frac{n_{1}^{1}}{C_{\mathrm{Tot}}}}{q\left(1-\frac{q^{2}}{C_{\mathrm{Tot}}} m_{1}^{1} \alpha_{\mathrm{D}}\right)} \\
V_{\mathrm{TH}}=V_{\mathrm{GS}}-V_{\mathrm{DSsat}}
\end{array}\right. \\
& \left\{\begin{array}{l}
U_{\mathrm{SCF}_{11,12}}=\frac{-q \alpha_{\mathrm{G}} V_{\mathrm{GS}}-q \alpha_{\mathrm{D}} V_{\mathrm{DS} 1,2}+\frac{q^{2}}{C_{\mathrm{Tot}}} n_{1}^{1}}{1-\frac{q^{2}}{C_{\mathrm{Tot}}} m_{1}^{1}} \\
U_{\mathrm{SCF}_{12,22}}=-q \alpha_{\mathrm{G}} V_{\mathrm{GS}}-q \alpha_{\mathrm{D}} V_{\mathrm{DS} 1,2} \\
I_{1,2}=I_{0} \times \operatorname{Ln}\left(1+\exp \left(\frac{E_{\mathrm{F}}-U_{\mathrm{SCF}_{1,2}}}{K_{\mathrm{B}} T}\right)\right. \\
V_{\mathrm{A}}=\frac{I_{1} \times\left(V_{\mathrm{DS} 1}-V_{\mathrm{DSsat}}\right)-I_{2} \times\left(V_{\mathrm{DS} 1}+0.01-V_{\mathrm{DSsat}}\right)}{I_{2}-I_{1}} \\
k=\frac{\left(I_{1}-I_{2}\right) \times V_{\mathrm{A}}}{V_{\text {DSsat }}^{2} \times 0.01} .
\end{array}\right.
\end{aligned}
$$

The complete model is constructed by inserting these parameters into the previous model, which is [9],

$$
I_{\mathrm{DS}}=\left\{\begin{array}{c}
k\left[A V_{\mathrm{DS}} V_{\mathrm{DSsat}}-B V_{\mathrm{DS}}^{2}\right] \quad \text { for } V_{\mathrm{GS}} \geq V_{\mathrm{TH}} \\
\text { and } V_{\mathrm{DS}} \leq V_{\mathrm{DSsat}} \\
k V_{D S s a t}^{2}\left[1+\frac{\left.V_{D S}-V_{D S s a t}\right]}{V_{A}} \quad \text { for } V_{\mathrm{GS}} \geq V_{\mathrm{TH}}\right. \\
\text { and } V_{\mathrm{DS}}>V_{\mathrm{DSsat}}
\end{array}\right.
$$




\section{Simulation results}

In order to evaluate our model accuracy at different bias and physical parameter conditions, we have compared model output at different CNT's diameters $(d)$, oxide's thickness $\left(t_{\mathrm{ox}}\right)$, source Fermi energy $\left(E_{\mathrm{F}}\right)$, and gate-source voltages at different $V_{\mathrm{DS}}$ which are shown in figures. At all figures, value of gate-source voltage is $1 \mathrm{~V}$. We have compared the results obtained from our model with those of FETToy.

The illustrations in Fig. 2 are a comparison between the proposed and FETToy models made for various CNTFETs with different values of carbon nanotube diameters. The CNT diameters were selected in the range of $0.5 \mathrm{~nm} \leq d \leq 1.5 \mathrm{~nm}$, a range for practically used CNTs. The values of the parameters used in this comparison are the same as those considered in [9], i.e., $T=300 \mathrm{~K}$, $C_{\text {Tot }}=172 \quad \mathrm{aF}-\mu \mathrm{m}-1, \quad \alpha_{\mathrm{G}}=0.93, \quad \alpha_{\mathrm{D}}=0.035, \quad \varepsilon_{r}(-$ $\left.\mathrm{SiO}_{2}\right)=3.9, t_{\mathrm{ox}}=1.5 \mathrm{~nm}$, and $E_{\mathrm{F}}=-0.32 \mathrm{eV}$. The figure was obtained under constant $V_{\mathrm{GS}}=0.5 \mathrm{~V}$. As seen from the figure, the dependency of $I_{\mathrm{DS}}$ to CNT diameter can be appropriately modeled by the proposed model. The drain-source current is an increasing function of $d$, because of decreasing in band-gap energy and increasing in CNT density of states.

An evaluation is carried out to verify the validity of the model in which the gate-source voltage changes. Such assessment is depicted in Fig. 3. In this figure, $V_{\mathrm{GS}}$ varied from 0.4 to $0.6 \mathrm{~V}$, while $d=1 \mathrm{~nm}$ and the values for other parameters are the same as used for Fig. 2. The current is raised by increasing the $V_{\mathrm{GS}}$ due to lower channel potential barrier seen by the carriers. The results show that the proposed model could accurately predict such a relationship.

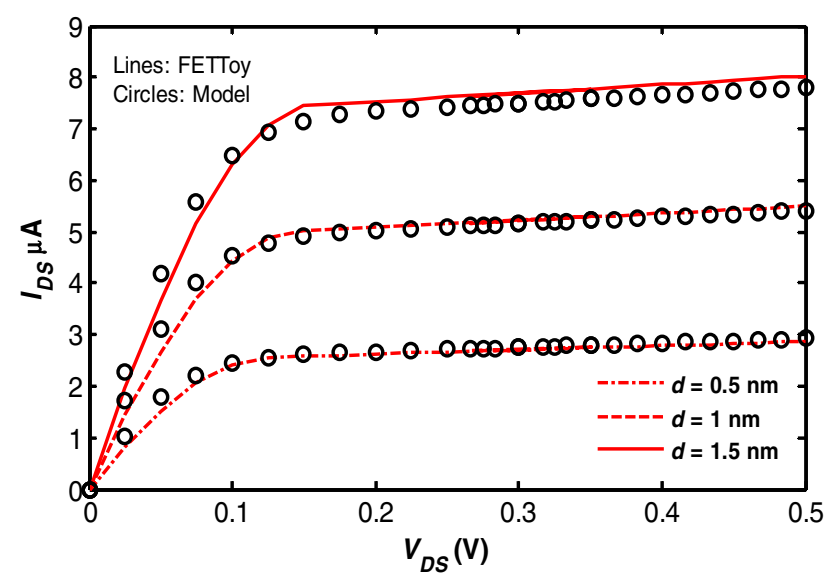

Fig. 2 Comparison of $I_{\mathrm{DS}}-V_{\mathrm{DS}}$ characteristics obtained by proposed model and FETToy for CNTFETs with various values of CNT diameters, under constant $V_{\mathrm{GS}}=0.5 \mathrm{~V}$. The values of other parameters are: $T=300 \mathrm{~K}, \quad C_{\mathrm{Tot}}=172 \mathrm{aF}-\mu \mathrm{m}-1, \quad \alpha_{\mathrm{G}}=0.93$, $\alpha_{\mathrm{D}}=0.035, \varepsilon_{r}\left(\mathrm{SiO}_{2}\right)=3.9, t_{\mathrm{ox}}=1.5 \mathrm{~nm}$, and $E_{\mathrm{F}}=-0.32 \mathrm{eV}$

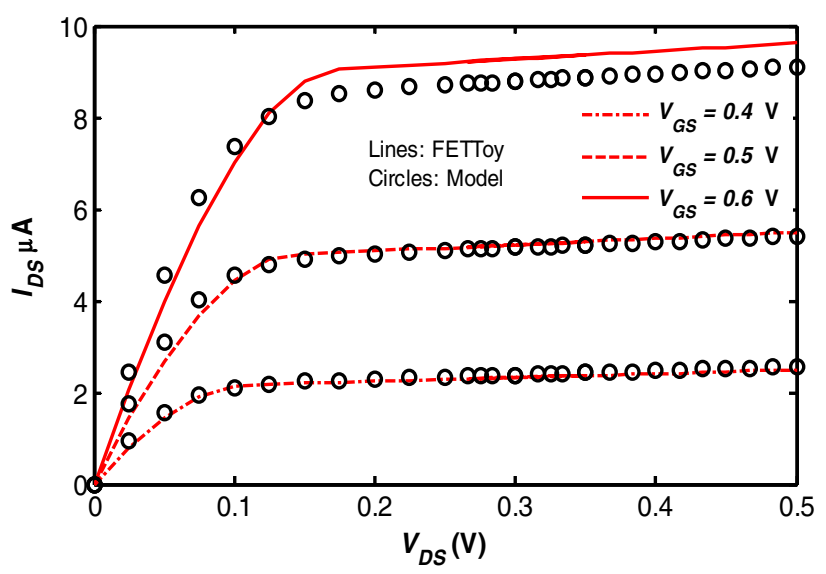

Fig. 3 Comparison of $I_{\mathrm{DS}}-V_{\mathrm{DS}}$ characteristics obtained by proposed model and FETToy for CNTFETs with various values of $V_{\mathrm{GS}}$. The CNT diameter $d=1 \mathrm{~nm}$, and the values of remaining parameters are the same as those in Fig. 2

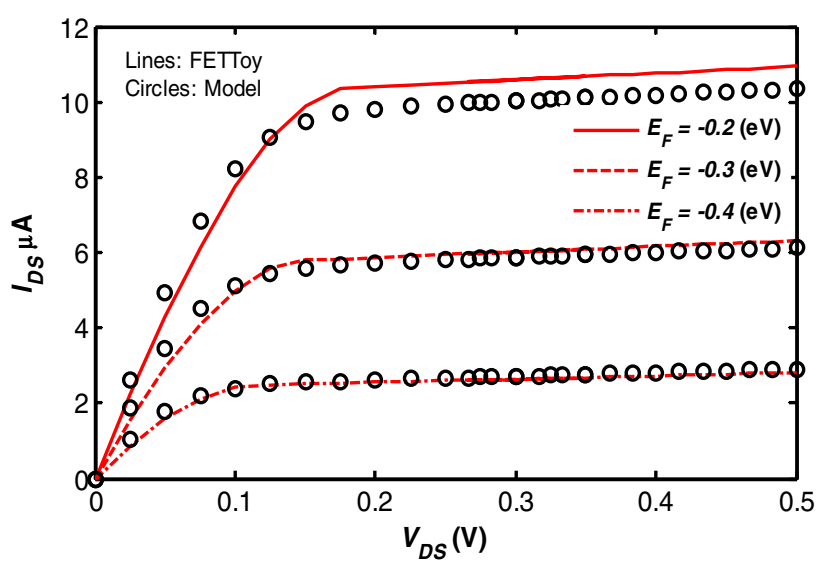

Fig. 4 Comparison of $I_{\mathrm{DS}}-V_{\mathrm{DS}}$ characteristics obtained by proposed model and FETToy for CNTFETs with various values of $E_{\mathrm{F}}$. The CNT diameter is $d=1 \mathrm{~nm}$, and the values of remaining parameters are the same as those in Fig. 2

The accuracy of the proposed model for different values of the source Fermi levels is validated in Fig. 4. This assessment is done for $E_{F}$ in the range of $-0.2 \mathrm{eV} \leq E_{F} \leq-0.4 \mathrm{eV}$, while $d=1 \mathrm{~nm}$ and other parameters are kept the same as those in Fig. 2. The figure shows that the results obtained from the proposed model are in excellent agreement with those of FETToy.

A further comparison is made by varying relative oxide permittivity, $\varepsilon_{\mathrm{r}}$, while $d=1 \mathrm{~nm}$ and keeping the remaining parameters the same as those used for Fig. 2. Results of such a comparison, reported in Fig. 5, reveal excellent modeling capabilities of the proposed model in these situations. The current increases as the oxide permittivity increases, as a result of enhancing of the gate contact control on the channel potential by increasing the gate 


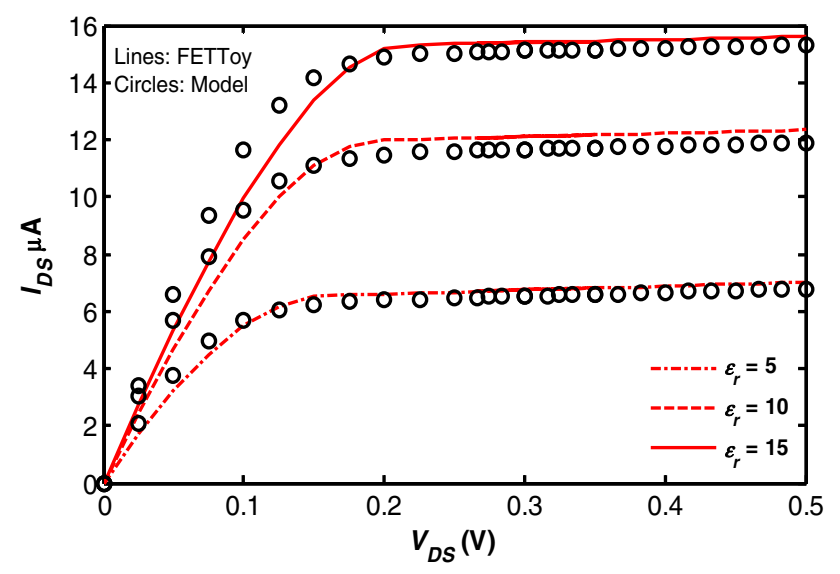

Fig. 5 Comparison of $I_{\mathrm{DS}}-V_{\mathrm{DS}}$ characteristics obtained by proposed model and FETToy for CNTFETs with various values of $\varepsilon_{r}$. The CNT diameter is $d=1 \mathrm{~nm}$, and the values of remaining parameters are the same as those in Fig. 2

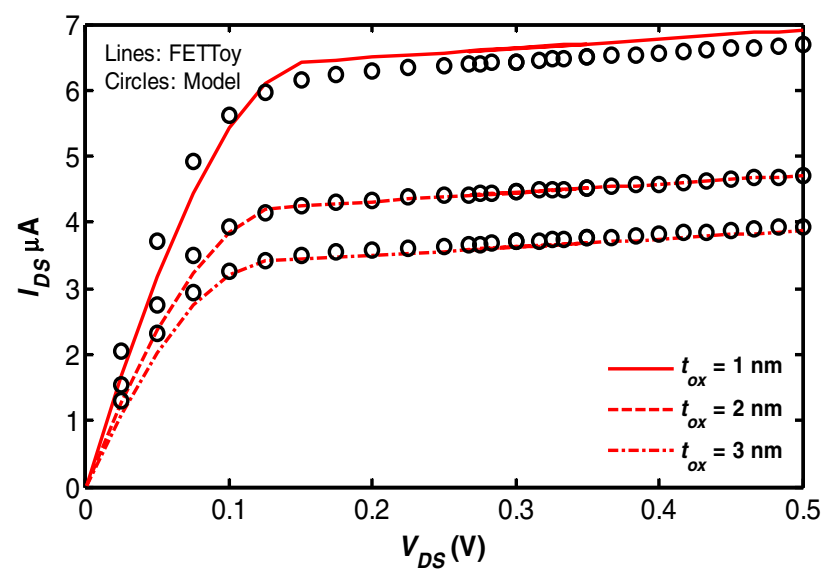

Fig. 6 Comparison of $I_{\mathrm{DS}}-V_{\mathrm{DS}}$ characteristics obtained by proposed model and FETToy for CNTFETs with various values of $t_{\mathrm{ox}}$. The CNT diameter is $d=1 \mathrm{~nm}$, and the values of remaining parameters are the same as those in Fig. 2

capacitance. As the figure shows, this relation could be modeled by the proposed model with sufficient accuracy.

Finally, we have made a comparison of the outputs of the proposed model with those of FETToy for a variety of oxide thickness, while $d=1 \mathrm{~nm}$ and the remaining parameters were kept the same as those of Fig. 2. Figure 6 illustrates the simulation results of both models for aforementioned CNTFETs. As is clear from the figure, there is a good agreement between the results of proposed model and those of FETToy. Decreasing the gate oxide thickness improves the gate control on the channel. Therefore, the drain current is a decreasing function of $t_{o x}$. The accuracy of the proposed model for varying $t_{o x}$ is demonstrated by this evaluation.

\section{Conclusion}

The model based on neural space mapping is one of the models which has been proposed to model the MOSFET transistors based on carbon nanotubes. In this model, three neural networks were used for modifying three parameters of threshold voltage, Early voltage, and constant $k$ of a coarse model, which was a modified "level 1" MOSFET model in SPICE. The main disadvantage of this model is the existence of three neural networks, which is an undesired property for implementation in commercial circuit simulators such as SPICE. In the present work, three analytical relations were presented to replace these neural networks. Therefore, the neural space model was converted into a mathematical space model. Some comparisons were done between the $I_{\mathrm{DS}}-V_{\mathrm{DS}}$ characteristics of the proposed model and the main model by variation of nanotube diameter, oxide thickness, oxide permittivity, source Fermi level, and gate-source voltage. The results of these comparisons showed that the proposed model had reasonable accuracy at different biases and for different values of physical parameters of the transistor.

Open Access This article is distributed under the terms of the Creative Commons Attribution 4.0 International License (http://crea tivecommons.org/licenses/by/4.0/), which permits unrestricted use, distribution, and reproduction in any medium, provided you give appropriate credit to the original author(s) and the source, provide a link to the Creative Commons license, and indicate if changes were made.

\section{References}

1. Hashempour, H., Lombardi, F.: Device Model for Ballistic CNFETs Using the First Conducting Band. IEEE Des. Test Comput. 25, 178-186 (2008)

2. Chek, D.C.Y., Tan, M.L.P., Ahmadi, M.T., Ismail, R., Arora, V.K.: Analytical modeling of high performance single-walled carbon nanotube field-effect-transistor. Microelectron. J. 41, 579-584 (2010)

3. Yousefi, R., Shabani, M.: A model for carbon nanotube FETs in the ballistic limit. Microelectron. J. 42, 1299-1304 (2011)

4. Kazmierski, T.J., Al-Hashimi, B.M., Ashburn, P.: Numerically Efficient Modeling of CNT Transistors With Ballistic and Nonballistic Effects for Circuit Simulation. IEEE Trans. Nanotechnol. 9, 99-107 (2010)

5. Luo, J., Wei, L., Lee, C.-S., Franklin, A.D., Guan, X., Pop, E., Antoniadis, D.A., Wong, H.-S.P., Member, S.S., Abstract, A.: Compact model for carbon nanotube field-effect transistors including nonidealities and calibrated with experimental data down to 9-nm gate length. IEEE Trans. Electron Devices 60, 1834-1843 (2013)

6. McEuen, P.L., Fuhrer, M.S.: Single-walled carbon nanotube electronics. IEEE Trans. Nanotechnol. 1, 78-85 (2002)

7. Rahman, A., Datta, S., Lundstrom, M.S.: Theory of ballistic nanotransistors. IEEE Trans. Electron Devices 50, 1853-1864 (2003) 
8. Kazmierski, T.J., Zhou, D., Al-Hashimi, B.M.: Efficient circuitlevel modelling of ballistic CNT using piecewise non-linear approximation of mobile charge density. DATE08. pp. 146-151 (2008)

9. Yousefi, R., Saghafi, K., Moravvej-Farshi, M.K.: Application of neural space mapping for modeling ballistic carbon nanotube transistors. Iran. J. Electr. Electron. Eng. 6, 70-76 (2010)

10. Hayati, M., Rezaei, A., Seifi, M.: CNT-MOSFET modeling based on artificial neural network: application to simulation of nanoscale circuits. Solid State Electron. 54, 52-57 (2010)
11. Frégonèse, S., Cazin, H., Goguet, J., Maneux, C., Zimmer, T., Bourgoin, J., Dollfus, P., Galdin-retailleau, S.: Computationally Efficient Physics-Based Compact CNTFET Model for Circuit Design. 55, 1317-1327 (2008)

12. Zhang, Q.J., Gupta, K.C.: Neural network and space mapping technologies. IEEE MTT-S International Microwave Symposium Digest, 2005. pp. 1595-1595. IEEE (2005)

13. Tsividis, Y.: Operational modeling of the MOS transistor. McGraw-Hill, New York (1999) 